\title{
Aplicación de técnicas de corrección e intervención logopédica para la enseñanza de la articulación del fonema vibrante múltiple /r/ a estudiantes anglófonos de ELE
}

\author{
Belén Reyes Morente. Universidad de Granada
}

Recepción: 29/06/2018| Aceptado: 14/05/2019

Correspondencia a través de ORCID: Belén Reyes Morente

iD 0000-0003-0429-7940

Citar: Reyes Morente, B (2019). Aplicación de técnicas de corrección e intervención logopédica para la enseñanza de la articulación del fonema vibrante múltiple /r/ a estudiantes anglófonos de ELE. ReiDoCrea - Monográfico sobre Perspectivas transnacionales en la enseñanza de lenguas, 8(3), 48-59.

\begin{abstract}
Resumen: Dentro de la enseñanza del español como lengua extranjera no se han contemplado los puntos en común que pueden existir entre la adquisición de una L2 -en concreto, los procesos lingüísticos presentes en la llamada interlengua- y la rehabilitación en un trastorno del lenguaje, en otras palabras, la reeducación de la L1. De hecho, las estrategias de corrección de dislalias funcionales, es decir, aquellas cuya etiología no se corresponde con ningún trastorno físico conocido, no distan mucho de la corrección fonética de determinadas articulaciones que pueden verse afectadas, por ejemplo, por la interferencia de la L1. Por ello, esta investigación va a tratar de ver las similitudes de ambos ámbitos en el campo de la fonética y de la ortofonía, más concretamente se va a centrar en la realización problemática de la vibrante múltiple en alumnado anglófono. De esta manera, se va a comprobar si los alumnos mejoran la pronunciación de dicho fonema adaptando al aula de idiomas las técnicas empleadas por los terapeutas del lenguaje.
\end{abstract}

Palabras clave: Fonética | Trastornos del lenguaje

Speech pathology techniques to improve Spanish as L2 pronunciation for English native speakers

Abstract: The current methods for teaching Spanish do not envisage the potential common points between the learning process of a second language and speech therapy for a language disorder. In fact, the intervention strategies for functional dyslalia (those that do not correspond to any known physical damage or disorder), do not diverge much from the phonetics' correction that a Spanish Language teacher uses to explain certain phonemes. Therefore, this research aims to focus on seeking the similarities between the phonetics and the speech therapy, stressing the rolled ' $R$ ' pronunciation in students whose first language is English. Thus, it is tested if students show an improvement of their pronunciation by implementing speech therapists' techniques.

Keywords: Phonetics | Speech Pathology

\section{Introducción}

El presente trabajo está enfocado en la enseñanza de la pronunciación en el aula de ELE siguiendo una nueva metodología. Se trata de un método que tiene como base las técnicas de intervención logopédica que utilizan los terapeutas del lenguaje para rehabilitar a los pacientes, pero en este caso adaptadas al aula de idiomas.

En esta investigación se aúnan dos disciplinas que, aunque se han considerado siempre de ámbitos de conocimiento diferentes, tienen mucho en común. Lingüística y Logopedia, aun con ciertas diferencias, comparten un objeto de estudio es tan cercano que a veces se confunde. A pesar de esta proximidad, no todo el mundo es consciente de esta cercanía entre disciplinas. Si tomamos en consideración la opinión de Garayzábal (2005) esto se debe a que la idea que se tiene sobre la Lingüística es que solo se encarga de la gramática y de la corrección, desde una perspectiva fuertemente normativa, pero esta idea poco tiene que ver con la realidad. Nuestra disciplina cada vez está más relacionada con otros campos de conocimiento y tiene una vertiente más 
aplicada, más dedicada a la resolución de problemas reales. Tomando como referencia las palabras de Garayzábal (2005, p.174):

[...] orientación más aplicada de estas mismas investigaciones; en fin, abriéndonos a un presente en el que prima lo resolutivo, en el que el mercado demanda soluciones a problemas reales; dando paso a una lingüística "joven".

Así pues, lo que no está dando a entender la autora es que hay que abrir los horizontes de la disciplina para dar lugar a otro tipo de investigaciones, en este caso en el campo de la Lingüística Aplicada. Por ello, no nos debe extrañar ver cómo dos disciplinas como la Lingüística y la Logopedia trabajan juntas hasta el punto de dar a luz una nueva área de estudio resultado de esta interdisciplinariedad: la Lingüística clínica. Se trata de una disciplina reciente y, por lo tanto, inmersa en un proceso de construcción y delimitación de objetivos y metodologías propias.

Lo que queremos transmitir es que nosotros abogamos por la multidisciplinariedad. El hecho de que dos disciplinas se complementen hace que los profesionales se enriquezcan mutuamente con otros procedimientos, otros conocimientos, etc. En esta investigación estamos siendo multidisciplinares ya que hemos usado (aunque no exclusivamente) las técnicas que utilizan los logopedas, no los lingüistas, para abordar un problema de fonética articulatoria y pronunciación; por lo tanto, estamos enriqueciéndonos de "otra" disciplina. Como anteriormente se ha dicho y como mantiene Garayzábal (2005) la Lingüística no se debe encasillar, debe ampliar sus horizontes de actuación y nutrirse de otros campos de conocimiento. Asimismo, teniendo siempre en mente la idea de que los antiguos métodos de pronunciación no han funcionado, debemos explorar más allá para el beneficio de nuestros alumnos. Además de que en nuestra propuesta los alumnos no aprenden solo pronunciación, sino que también introducimos contenidos lingüísticos y culturales.

\section{El lugar de la pronunciación en la clase de ELE}

La pronunciación es una parte fundamental del uso de la lengua y, por lo tanto, tenemos que integrarla en nuestras clases de español para enseñarla a los alumnos, pero esta nunca ha ocupado un lugar fijo dentro de la enseñanza de lenguas. Según Iruela (2004, p.15): «la pronunciación no ha seguido el mismo camino que otras áreas de la enseñanza comunicativa de lenguas, como la gramática o la expresión escrita». Como mantiene Bartolí (2005) el lugar de la pronunciación ha ido dependiendo del método que en aquel momento predominara. En la actualidad el llamado enfoque comunicativo prevalece en casi todos los ámbitos de la enseñanza de lenguas y como dice Usó (2008) desatiende la parte fónica. Es decir, aunque este enfoque preste mayor atención a las destrezas comunicativas a su vez está olvidando integrar la pronunciación en el habla. Como mantiene Padilla (2006) en el enfoque comunicativo se considera que los alumnos adquieren una competencia fónica aceptable a través de la interacción con nativos, como por ejemplo su profesor; pero si tomamos esto como cierto, podemos hacer que nuestros alumnos arrastren problemas a la hora de pronunciar que les repercutan en su manera de comunicarse con los demás, creándoles inseguridad a la hora de hablar. Por lo tanto, como dice Usó (2008) este enfoque desatiende la parte fónica.

Según varios autores, Usó (2008) y Bartolí (2005), los problemas de no otorgar un tratamiento específico a la pronunciación dentro de las aulas de idiomas tienen su base en ciertos prejuicios sobre la enseñanza de esta. La primera idea negativa sobre esto, y la más extendida, es que para enseñar pronunciación hay que ser experto en fonética y fonología y, a consecuencia de ello, todos los profesores de ELE tendrían que tener una formación específica en estos ámbitos de la lingüística. Como apunta Bartolí (2005) 
la fonética es el campo de conocimiento que estudia los sonidos y, como se ha comentado antes, no tiene mucho en común con la definición de pronunciación otorgada. Por ello, hay que desterrar aquellos prejuicios que creen que para enseñar pronunciación hay que ser fonetista, los alumnos no necesitan hacer transcripciones ni teorizar sobre ello, sino que lo que quieren es conseguir una buena pronunciación. Sí que es cierto, como defiende Bartolí (2005) que, en lo que respecta a los profesores, para enseñar corrección fonética sí deben conocer algunos aspectos básicos de fonética, pero ello no implica que deban ser especialistas. La segunda idea falsa sobre esto es que, como mantiene Usó (2008) el español es una lengua "fonética", es decir, no hay distancia entre la ortografía y la pronunciación. Bien es cierto que hay menos distancia que en otras lenguas, como por ejemplo el francés o el catalán, pero esto no implica que ya no se tenga que enseñar pronunciación, ya que a pesar de ser una lengua «fácil» existen ciertos fonemas problemáticos. Ligada a esta idea, Usó (2008) citando a Carbó et al. (2003) ${ }^{1}$, mantiene que tan solo con que el alumno esté en inmersión lingüística ya va a ser capaz de adquirir una competencia fónica y que, si este no está en el país en el que se habla dicha lengua, este aprendizaje no va a ser fructífero. El último prejuicio tiene también como base la formación de los alumnos: como explica Usó (2008) está muy extendida la idea de que el alumno para recibir una clase de pronunciación debe tener una buena base gramatical y léxica, pero de nuevo estamos olvidando enseñar a nuestros alumnos a pronunciar.

De esta manera, como estos prejuicios respecto a la pronunciación han estado vigentes durante mucho tiempo, y algunos de ellos lo siguen estando, esto ha tenido consecuencias que se han visto reflejadas en las aulas de idiomas siendo la más importante la relacionada con los materiales didácticos utilizados.

Para conseguir integrar la pronunciación en las aulas de idiomas deberíamos tener en cuenta varios factores. Como mantiene Llisterri (2003) si queremos abordar el tema de la pronunciación en nuestras aulas debemos seguir una programación y, para ello, hay que seguir varios pasos: el primero de ellos, fijar los objetivos respecto a la norma que vamos a enseñar y cuánto debemos enseñar; el segundo, un análisis de la interlengua de los alumnos para prevenir errores. Nuestra investigación tiene su núcleo aquí puesto que está orientada a estudiantes anglófonos aprendices de español. Gracias al conocimiento de su lengua materna pudimos prever que tendrían problemas en el aprendizaje del fonema / $\mathrm{r} /$, dado que en su L1 este fonema no existe y al aprender la L2 -en el proceso de interlengua- este fonema sigue sin «aparecer». Como bien mantiene Llisterri (2003, p.95): «[...] los errores de pronunciación en una L2 se derivarán de los elementos de la L2 inexistentes en la L1 (por ejemplo [r] en el caso de un anglófono que aprenda español)». El tercer paso sería realizar una clasificación y jerarquía de dichos errores; finalmente, el cuarto paso: una planificación de la corrección en el aula. Consideramos que este esquema sigue un orden lógico y recomendable para poder integrar de forma satisfactoria la pronunciación en las aulas.

\section{La pronunciación y los trastornos del lenguaje}

Retomando la idea de que Lingüística y Logopedia comparten un objeto de estudio, revisando la historia de los métodos de enseñanza de la pronunciación se puede observar como estas disciplinas coinciden en varios aspectos. El primer punto de encuentro lo hallamos en lo que Bartolí (2005) reconoce como «corrección fonética». Esta rama de la fonética se encarga de ofrecer técnicas y métodos para la enseñanza

\footnotetext{
${ }^{1}$ Carbó, C., Llisterri, J., Machuca, M.J., De la Mota, C., Riera, M., Ríos, A. (2003). "Estándar oral y enseñanza de la pronunciación del español como primera lengua y como lengua extranjera", ELUA, Estudios de Lingüística de la Universidad de Alicante, 17: 161-180. http://liceu.uab.es/publicacions/carbo_et_al_EULA03.pdf
} 
de la pronunciación y la corrección de los problemas que los alumnos puedan tener. Así pues, como cita Bartolí: "La corrección fonética consiste en corregir los errores de pronunciación del alumno de una LE, como si fuese un hablante defectuoso de dicha lengua». Observamos como al alumno se le equipara con una persona con un trastorno del lenguaje, por lo que siguiendo esta afirmación sería lógico aplicar técnicas que utilizan los terapeutas del lenguaje para la «rehabilitación» de estos. Dentro de esta propia afirmación y de la perspectiva de la corrección fonética, nos encontramos con diferentes puntos de vista que se adoptan a la hora de corregir al alumno. Como cita Usó (2008, p.110): «perspectiva preceptiva, perspectiva terapéutica y perspectiva comunicativa.» De todas ellas, la que nos atañe es la perspectiva terapéutica puesto que trata al alumno como un paciente $y$, además, se señala que el alumno no es responsable de sus problemas de pronunciación sino que estos están relacionados con su L1 y la interlengua; así pues, como dice Usó (2008, p.110): «[...] el alumno no es responsable de su mala pronunciación sino su L1 y se lo debe tratar como a un paciente que sufre una "patología del habla"».

Tomando estas ideas como referencia, consideramos que a la hora de tratar un problema de pronunciación específico deberíamos adoptar la perspectiva terapéutica y, como se ha mencionado anteriormente, sería adecuado intentar aplicar ciertas técnicas usadas por los logopedas, siempre y cuando el problema en concreto se corresponda con un trastorno del lenguaje. En el caso de esta investigación, como nos hemos centrado en la realización problemática de la vibrante múltiple $/ r /$ en alumnado anglófono, que es consecuencia de la interferencia de su L1, hemos llegado a la conclusión de que este problema sí que tiene relación directa con un trastorno del lenguaje específico: la dislalia. Este trastorno del lenguaje se caracteriza por la mala articulación de algún fonema y, como apunta Pascual puede afectar a cualquier sonido consonántico o vocálico, a uno solo o a varios de ellos. Por ello, observamos que se basa en problemas de pronunciación o articulación, lo cual tiene correspondencia directa con nuestros alumnos de ELE y su pronunciación problemática de la vibrante múltiple.

Dentro de la dislalia encontramos que, como menciona Pascual, dependiendo de las causas que originan este trastorno se puede clasificar en: dislalia evolutiva, dislalia funcional, dislalia audiógena y dislalia orgánica. La que nos atañe es la dislalia funcional puesto que es aquella que, como apunta Pascual (2012), se muestra como un defecto en la articulación del lenguaje, pero sin que exista un problema físico en los órganos, o modificaciones en estos, por lo que su origen está en una incapacidad funcional. Esta dislalia es la que más se acerca a nuestros alumnos ya que estos no tienen problemas físicos ni modificaciones en los órganos, sino que presentan una mala articulación.

Como ya se ha mencionado anteriormente, la dislalia puede afectar a cualquier consonante o vocal. En el caso particular de la dislalia funcional, Pascual apunta (2012) que los errores más prototípicos de esta suelen ser: sustitución, omisión o deformación; y los fonemas más problemáticos son: $/ \mathrm{r} /, / \mathrm{k} /, / \mathrm{s} /$, /z/, y /t $/$ en el caso del español como L2. Así pues, identificamos que la vibrante múltiple es un fonema problemático en las personas que sufren dislalia funcional. Señala Pascual $(2012$, p.156) que los fonemas vibrantes son aquellos en los que: «[...] se dan una serie de interrupciones momentáneas durante la salida del aire fonador, producidas por el contacto entre el ápice de la lengua y los alvéolos [...]». Así pues, cada realización problemática de un fonema recibe un nombre y, el que se corresponde con el de la vibrante se denomina rotacismo, que se encuentra normalmente en los casos de dislalias funcionales.

De esta manera, nos parece claro que sí existe una relación directa entre un problema de articulación de la vibrante múltiple tanto por personas con un trastorno del lenguaje, dislalia, como por parte del alumnado anglófono estudiante de ELE. Por ello, 
consideramos que la aplicación de las técnicas logopédicas puede ayudar a nuestros alumnos para mejorar, o incluso a adquirir, la pronunciación de dicho fonema.

\section{Objetivos o hipótesis}

La hipótesis que nos planteamos es la siguiente: ¿Pueden ayudar las técnicas de intervención logopédica en la corrección de un fonema específico en alumnos de ELE?

El objetivo principal de la investigación es hacer una demostración empírica de que Lingüística y Logopedia tienen puntos en común y que, además, sobre esas coincidencias podemos trabajar diferentes aspectos. Relacionado con esto, el segundo objetivo que se plantea es que, tras demostrar la relación entre estas disciplinas, crear una unidad didáctica para llevarla a cabo en el aula de idiomas pero que tenga como base las técnicas logopédicas que ayuden a los alumnos a conseguir una pronunciación correcta del fonema vibrante.

\section{Métodos}

\section{Participantes}

Los participantes en el estudio fueron un grupo de estadounidenses cuyas edades oscilan entre los 19 y 20 años, dos hombres y ocho mujeres, que estaban realizando un curso de español en inmersión lingüística en el Centro de Lenguas Modernas de Granada. El curso en el que se realizó la investigación fue un curso de español como lengua extranjera centrado en la enseñanza de conceptos gramaticales. El nivel de los alumnos eran un nivel intermedio. Fueron varios los motivos por los que fueron seleccionados los alumnos. El primero de ellos fue que la secuencia didáctica requería que tuvieran un nivel intermedio del idioma y, el segundo de ellos, que su lengua materna fuera el inglés. El motivo para elegir hablantes anglófonos es que la realización problemática de la vibrante múltiple por parte de hablantes nativos de inglés se corresponde con un problema de interlengua, puesto que muchos presentan dificultades para diferenciar la vibrante simple de la múltiple. Esto es una consecuencia de la $r$ retrofleja de su lengua materna que se parece más a la $t$ de palabras inglesas como: better o butter. Esta $r$ se caracteriza por hacer una breve oclusión del ápice de la lengua contra los alvéolos, mientras que nuestra vibrante múltiple se caracteriza por hacer varias oclusiones contra los alvéolos.

\section{Instrumentos}

Los instrumentos utilizados para la puesta en práctica de la unidad didáctica son dos softwares de análisis articulatorio y acústico. El primero de ellos es Sounds of Speech que se trata de un programa creado por la Universidad de lowa que nos permite observar cómo es la articulación de un fonema en específico y, el segundo, es Speech Analyzer que es un software creado por el Summer Institute of Linguistics cuya función es el estudio del sonido desde el punto de vista físico. El motivo por el que se decide utilizar estos programas es porque, con el primero de ellos, los alumnos van a poder observar la posición de los órganos articulatorios para la realización del fonema y, con el segundo, se puede mostrar como es representada la vibrante múltiple y establecer comparaciones entre la onda de sonido de un nativo y un no nativo. 


\section{Resultados}

\section{Pretest}

Los resultados se obtienen en forma de espectograma y se pueden dividir en pretest y post test. En primer lugar, a los alumnos se les explica y se les muestra como es la estructura prototípica de la vibrante múltiple en español. Esta es muy especial puesto que las consonantes vibrantes, como mantiene Quilis (1999, p. 332), se caracterizan por su cualidad de interruptas. Hay que hacer hincapié en las cuatro oclusiones, o composiciones espectrográficas verticales como lo denomina Quilis (1999, p.334), que se observan en el espectrograma (señaladas con un cuadrado) ya que este programa es tan preciso que muestra hasta cuántos golpes da el ápice de la lengua contra el alveolo a la hora de pronunciar la vibrante múltiple

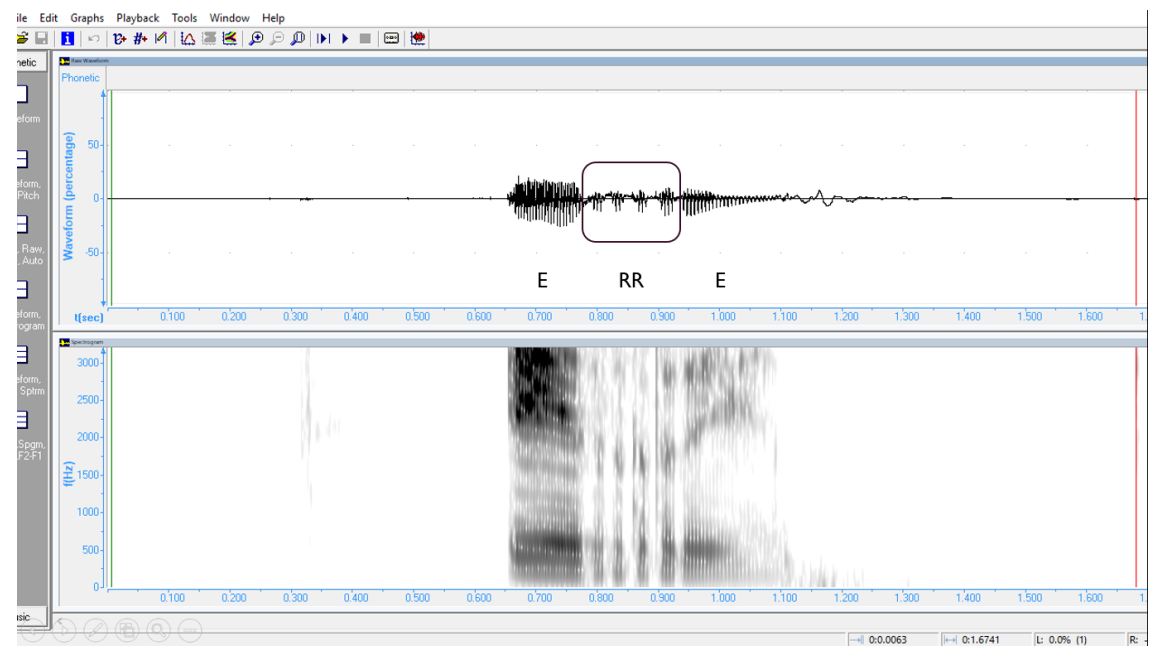

Figura 1. Imagen del programa Speech Analyzer que muestra la estructura del fonema.

En los espectogramas relativos al pretest, con la primera palabra que se trabaja <rosa> en la onda sonora de un nativo se observan dos oclusiones debido a que la vibrante múltiple está al principio; en los espectogramas de los alumnos antes del tratamiento la estructura de este fonema no aparece, sino que en su lugar hay una marca de sonoridad que se debe a que al ser una consonante sonora las cuerdas vocales vibran y el programa capta esa vibración. Por lo tanto, se puede concluir con el alumno no articula bien el sonido.

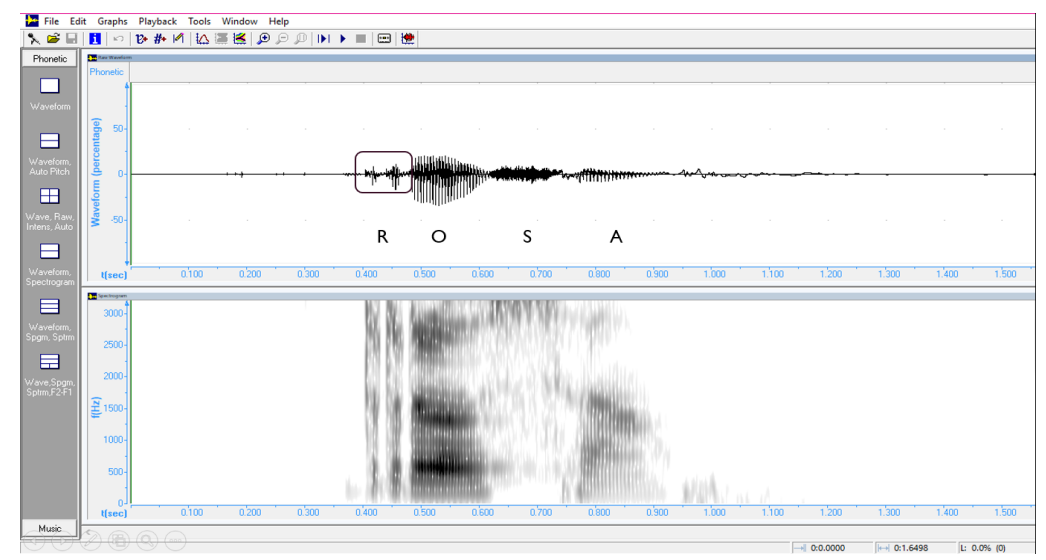

Figura 2. Imagen del programa Speech Analyzer que muestra la onda sonora de la palabra <rosa> por parte de un nativo. 


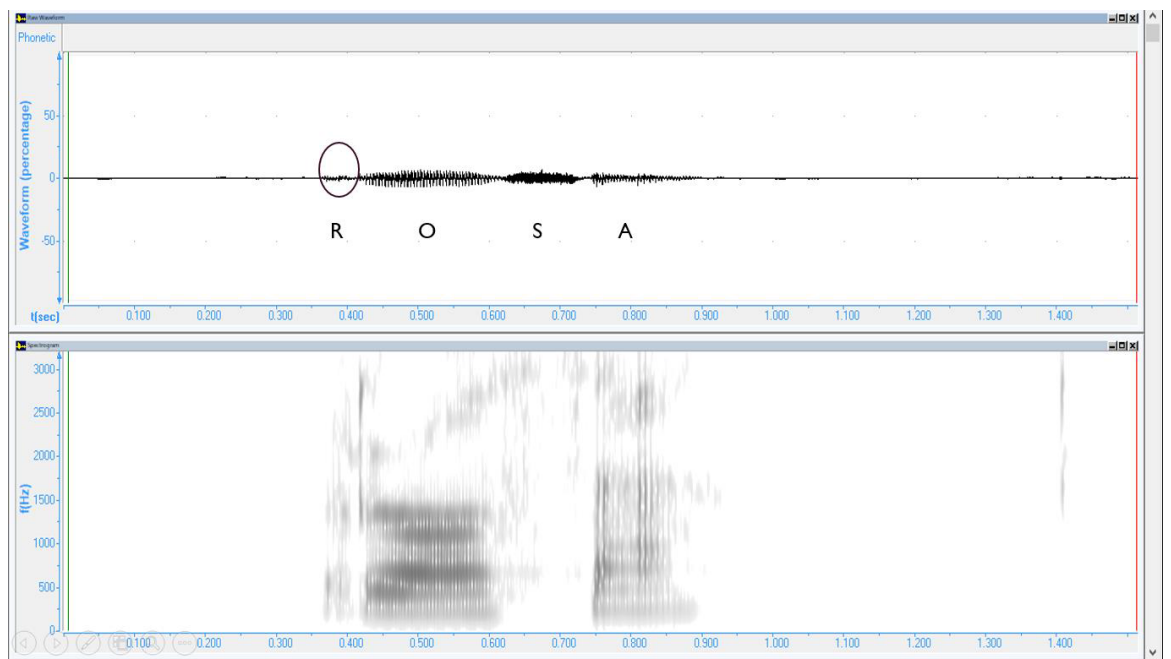

Figura 3. Imagen del programa Speech Analyzer que muestra la onda sonora de la palabra <rosa> por parte de un alumno en el pretest.

Siguiendo con el pretest, la segunda palabra con la que se trabaja es <torre> la cual, al estar en posición intervocálica, en el espectograma aparecen tres oclusiones, es decir, que el ápice de la lengua ha golpeado tres veces el alveolo. En las grabaciones de los alumnos analizadas con el programa se observa que la estructura del fonema no aparece, de nuevo encontramos una marca de sonoridad que se aleja de lo que se considera la estructura de la vibrante.

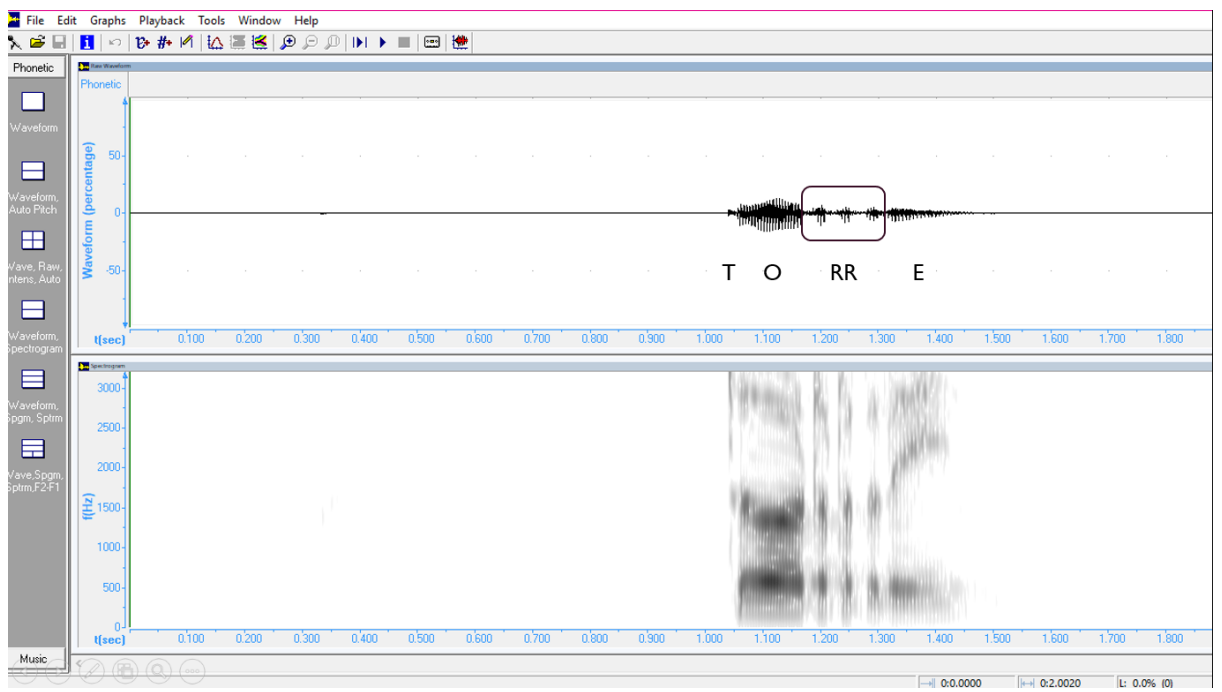

Figura 4. Imagen del programa Speech Analyzer que muestra la onda sonora de la palabra <torre> por parte de un nativo. 


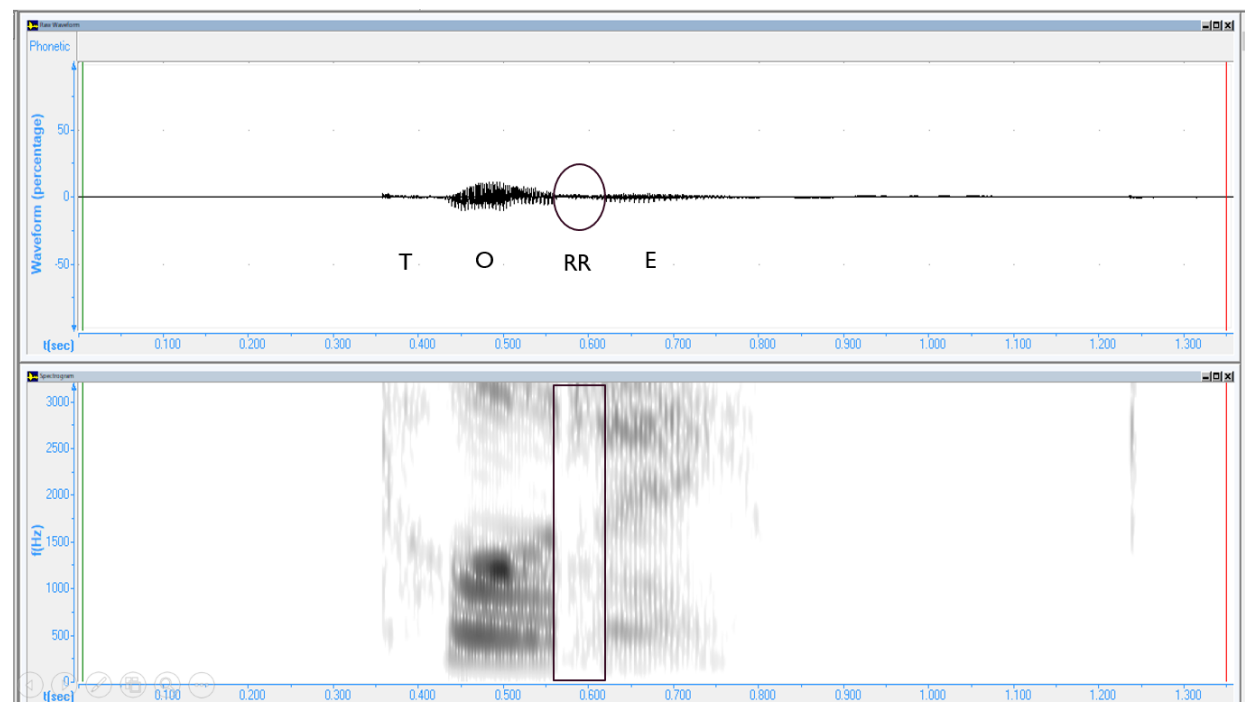

Figura 5. Imagen del programa Speech Analyzer que muestra la onda sonora de la palabra <torre> por parte de un alumno en el pretest.

La última palabra con la que se trabajo fue <sonreír> cuya vibrante múltiple se ve representada en el espectograma con dos oclusiones. Los resultados de los alumnos con respecto a esta palabra están bastante alejados de lo que se considera una buena pronunciación, puesto que no aparece la estructura de la vibrante, las dos oclusiones, sino que de nuevo hay una marca de sonoridad.

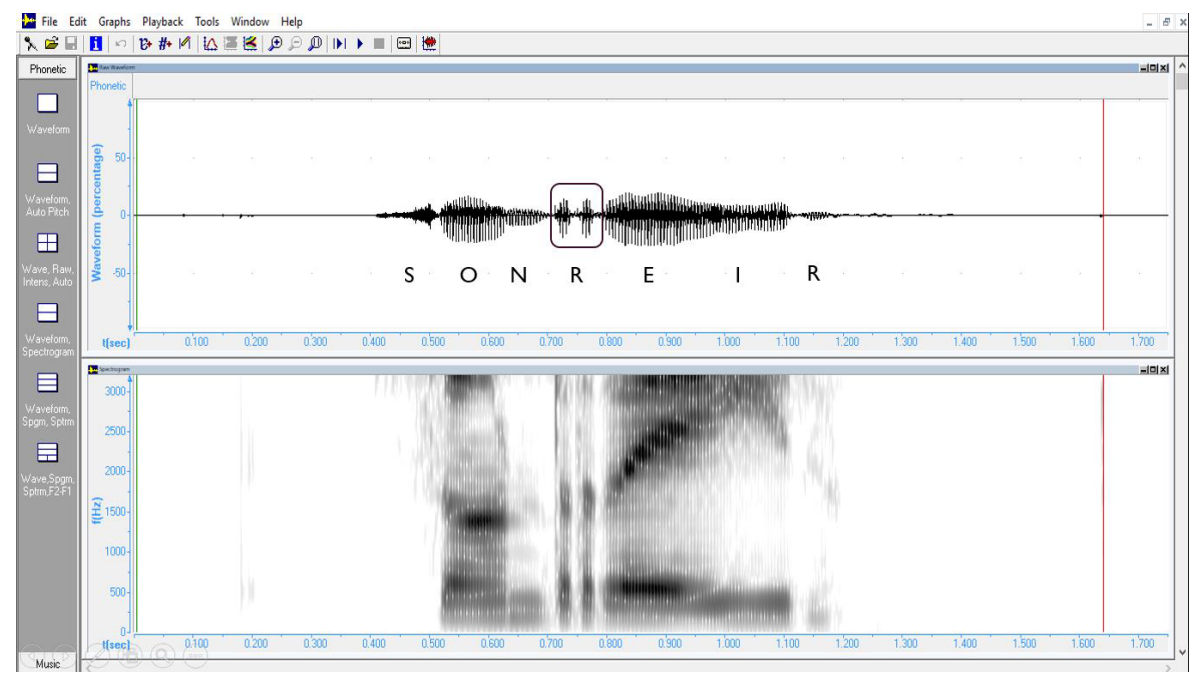

Figura 6. Imagen del programa Speech Analyzer que muestra la onda sonora de la palabra <sonreír> por parte de un nativo.

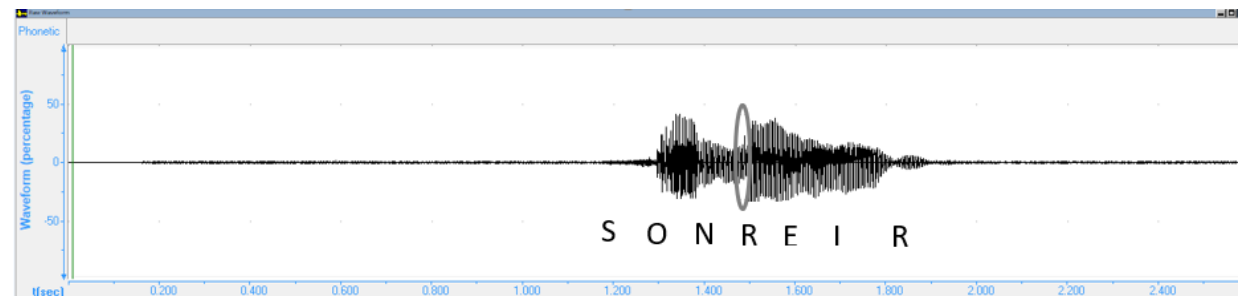

Figura 7. Imagen del programa Speech Analyzer que muestra la onda sonora de la palabra <sonreír > por parte de un alumno en el pretest. 


\section{Post test}

Tras la puesta en práctica de la secuencia didáctica con las técnicas logopédicas como base los resultados son bien diferentes. En el post test, con respecto a la palabra <rosa> encontramos que la estructura de la vibrante múltiple aparece marcada, es decir, se muestran tres oclusiones. Lo habitual serían dos, pero esto se debe al sobreesfuerzo del alumno para lograr la pronunciación.

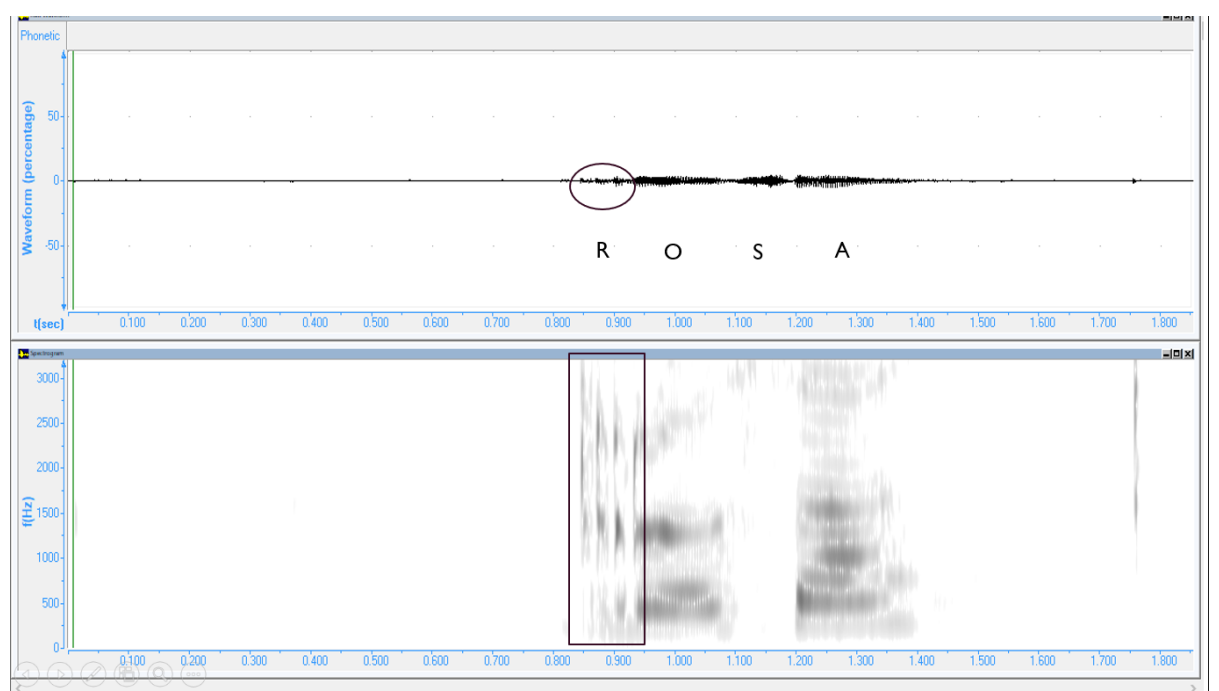

Figura 8. Imagen del programa Speech Analyzer que muestra la onda sonora de la palabra <rosa> por parte de un alumno en el post test.

Con la palabra <torre>, la más sorprendente de todas, en los espectogramas de algunos alumnos se puede observar perfectamente las tres oclusiones que caracterizan al fonema; incluso, en algunas ondas de sonido se pueden ver hasta cuatro oclusiones, consecuencia del sobreesfuerzo del alumno para lograr la pronunciación adecuada.

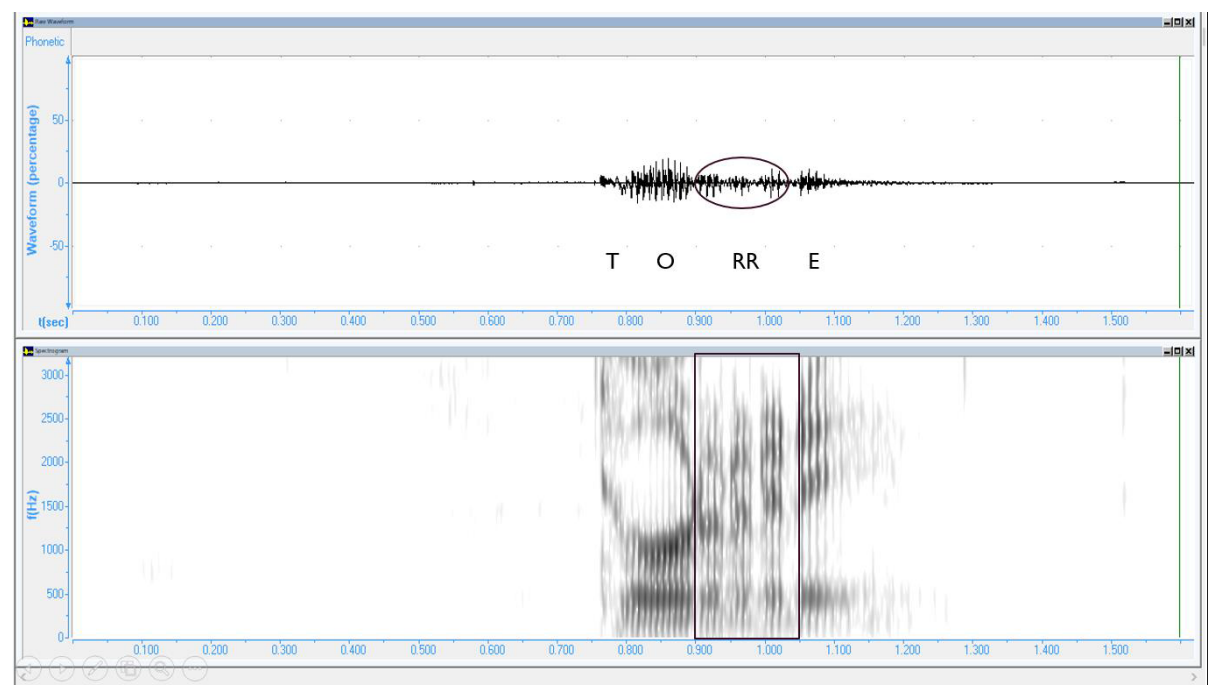

Figura 9. Imagen del programa Speech Analyzer que muestra la onda sonora de la palabra <torre> por parte de un alumno en el post test. 
Por último, con la última palabra <sonreír> se observa que hay algunos alumnos que consiguen alcanzar una buena pronunciación de la palabra porque se observa las dos oclusiones características de la palabra y, como anteriormente se ha mencionado, en algunos casos se ven hasta tres oclusiones debido de nuevo al sobreesfuerzo del alumno.

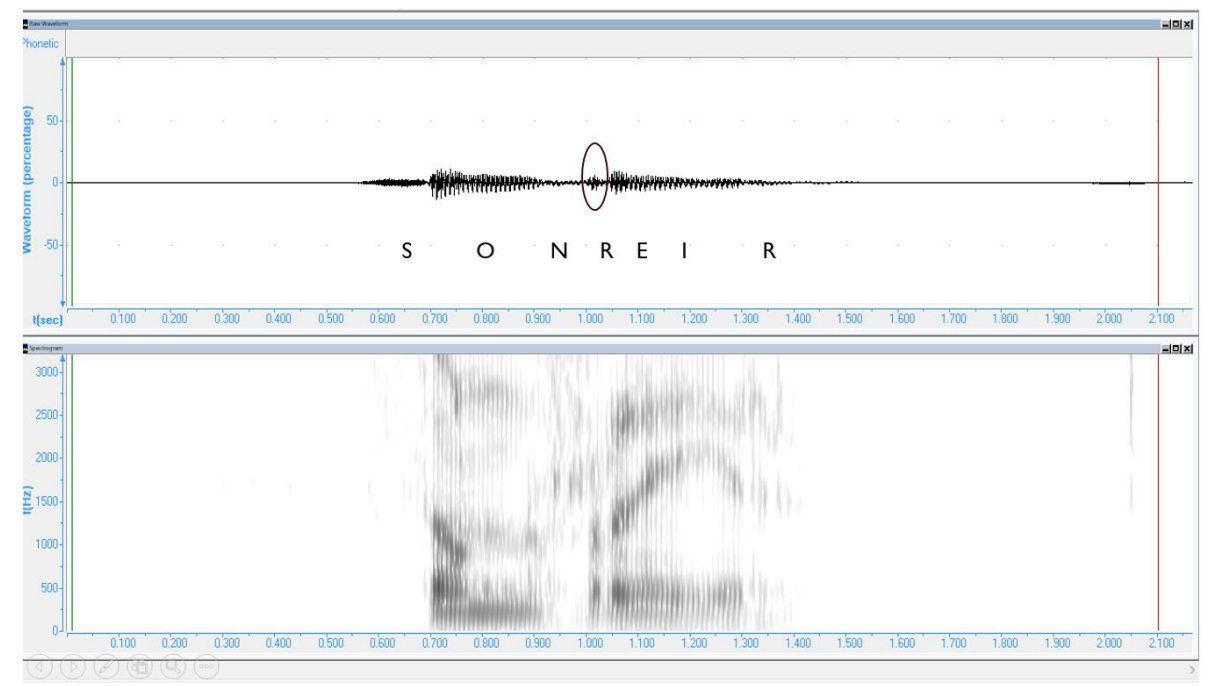

Figura 10. Imagen del programa Speech Analyzer que muestra la onda sonora de la palabra <torre> por parte de un alumno en el post test.

Así pues, la diferencia entre los resultados del pretest y el post test es notable a consecuencia de la efectividad de la puesta en práctica de las técnicas logopédicas adaptadas al aula de idiomas.

\section{Discusión}

La principal hipótesis de este trabajo era si dos disciplinas que se han considerado tradicionalmente opuestas pueden trabajar juntas, en ello reside la principal innovación de esta investigación. La pretensión de ser multidisciplinarios y querer aunar ambas disciplinas se ha valorado siempre en base a las necesidades de los alumnos, es decir, cuando se alcanza un cierto nivel de lengua se espera que, además de hablar de forma fluida, tengan una buena pronunciación. Por lo tanto, revisando los métodos de enseñanza y la historia de la pronunciación se puede observar que no se ha encontrado el método adecuado. Así pues, observando los resultados obtenidos se puede concluir que esta metodología además de ser innovadora es efectiva.

Como toda investigación, es obvio que hay limitaciones y fortalezas del estudio. Como ya se ha mencionado anteriormente, una de las fortalezas de esta investigación es el carácter innovador que posee. Por otra parte, las limitaciones del estudio son varias. En primer lugar, cuando se estudia en profundidad como es la terapia que aplican los logopedas para la rehabilitación de ciertos trastornos esta es muy extensa y consta de muchas sesiones. Así pues, una de las limitaciones que tuvo el estudio fue que solo se disponía de hora y media para realizar todo el tratamiento, por lo que se tuvieron que seleccionar los mejores ejercicios y los más efectivos pero, a consecuencia de ello, no fue posible observar la progresión de los alumnos si hubiéramos tenido más sesiones con ellos. Otra de las limitaciones de la investigación es que no se tuvieron en cuenta ciertas variables que pueden afectar a los resultados. Por ejemplo, el aprendizaje que han tenido los alumnos a lo largo de su carrera académica, si han tenido un profesor nativo o no; otra de estas variables puede ser si la inmersión lingüística ha influido en 
ellos, porque como se menciona anteriormente, con el método comunicativo se pretende que los alumnos adquieran una buena pronunciación solo con el hecho de estar en contacto con nativos.

Tras finalizar con el trabajo de fundamentación teórica y la puesta en práctica de la secuencia didáctica llegamos a varias conclusiones. La primera de ellas es que confirmamos la hipótesis planteada al principio, por lo que podemos decir que esta «nueva» metodología que tiene como base las técnicas logopédicas funcionan inicialmente, como se ha podido observar en los resultados.

En segundo lugar, consideramos que se ha demostrado que estas dos disciplinas, Lingüística y Logopedia, que se ha considerado que pertenecían a ámbitos del conocimiento diferentes pueden trabajar juntas. De esta manera, creemos que esto puede abrir un nuevo campo en la investigación de la adquisición de segundas lenguas.

Así pues, por último, destacar de nuevo la importancia de la multidisciplinariedad. Tomar como referencia dos disciplinas que se han considerado opuestas no ha sido sencillo, sobre todo por la carencia de bibliografía al respecto y, asimismo, por lo arriesgado de la investigación, ya que no sabíamos si iba a funcionar. Nosotros como profesionales consideramos que debemos ampliar nuestros campos de conocimiento y actuación ya que esto puede ser beneficioso para los alumnos.

En lo que respecta a la secuencia didáctica, debemos subrayar el efecto temporal puesto que se trata de una metodología rápida, entretenida para los alumnos y, lo más importante, efectiva.

\section{Referencias}

Bartolí, M. (2005). La pronunciación en la clase de lenguas extranjeras. Phonica, $1,1-27$.

Garayzábal-Heinze, E. y P. Otero (2005). Psicolingüística, Neurolingüística, Logopedia y Lingüística Clínica: Juntos sí, pero no revueltos. Filología y Lingüística, XXXI (1), 163-185.

Iruela, A. (2004). Adquisición y enseñanza de la pronunciación en lenguas extranjeras. (Tesis doctoral inédita dirigida por Francisco José Cantero Serena, Universitat de Barcelona) Recuperado de: https://dialnet.unirioja.es/servlet/tesis?codigo $=132073$

Llisterri, J. (2003). La enseñanza de la pronunciación. Revista del Instituto Cervantes en Italia, 4(1), 91-114.

Padilla, X. (septiembre, 2006). El lugar de la pronunciación en la clase de ELE. Comunicación presentada en Las destrezas orales en la enseñanza del español L2-LE: XVII Congreso Internacional de la Asociación del Español como lengua extranjera (ASELE), Logroño. Disponible en https://cvc.cervantes.es/ensenanza/biblioteca ele/asele/pdf/17/17 0871. pdf 
Pascual, P. (2012): La dislalia. Naturaleza, diagnóstico y rehabilitación. Madrid: Editorial CEPE.

Quilis, A. (1999): Tratado de fonología y fonéticas españolas. Madrid: Gredos.

Usó, L. (2008). La enseñanza de la pronunciación en LE: algunas consideraciones a tener en cuenta. Phonica, 4, 104-130. 